\title{
A Spectrum Sharing based Metering Infrastructure for Smart Grid Utilizing LTE and WiFi
}

\author{
Imtiaz Parvez, Arif I. Sarwat ${ }^{\Uparrow}$ \\ Department of Electrical and Computer Engineering, Florida International University, Miami, FL 33174
}

A R T I C L E I N F O

Article history:

Received: 12 December, 2018

Accepted: 09 February, 2019

Online: 15 March, 2019

Keywords:
$3.5 \mathrm{GHz}$
AMI
cognitive radio
LTE-U
CBRS
spectrum sharing
Internet of Things (IoT)
smart meter
licensed assisted access (LAA)
WiFi

\begin{abstract}
A B S T R A C T
In advanced metering infrastructure (AMI) of smart grid, WiFi is an appropriate choice for its bidirectional communication requirement to transmit data to the billing center. But, WiFi functions in the free spectrum bands and LTE also requires to use the same free bands for its network expansion being licensed spectrum is limited and expansive. LTE and WiFi can operate simultaneously in the $3.5 \mathrm{GHz}$ band (also known as citizen broadband radio service (CBRS)), which has large amount of free and clean spectrum. In this paper, we propose a smart grid metering infrastructure based on fixed duty cycled LTE and WiFi, where smart meters and its' data collectors (known as Access Point) use WiFi and LTE, respectively, for transferring data. Under a system level simulation environment, we investigated the LTE-WiFi coexistence performance in CBRS band considering a time division duplexing (TDD)-LTE associated with FTP traffic, and IEEE 802.11n (WiFi). The simulation performance demonstrates a good neighborhood coexistence between WiFi and LTE, which makes it a potential communication solution for the AMI.
\end{abstract}

\section{Introduction}

Smart grid is the advanced power and energy system that has been transformed from unidirectional power flow to bidirectional power flow. Moreover, it is employed with information communication technology among its entities for electricity supply to the consumers with reinforced control and efficiency [1, 2, 3, 4]. Advanced metering infrastructure (AMI) is an critical building block of smart grid as it builds communication bridge between metering data management service (MDMS) and consumer meters for consumption data transfer utilizing wireless networks [5, 6, 7, 8, 9]. The prominent communication standards for AMI are Zigbee, and $\mathrm{WiFi}$, that use public frequency bands e.g. $5 \mathrm{GHz}, 2.4 \mathrm{GHz}$, and $900 \mathrm{MHz}[10]$. Since unlicensed/public frequency bands will be shared, smart meters may need to coexist along with atypical techniques e.g. ZigBee and LTE in the same bands.

LTE is the long range broadband communication for exchanging voice and data [1,11]. For the advancement of requirement, LTE requires to accommodate machine-to-machine (M2M) communication in addi- tion to voice communication. Moreover, to satisfy the exponential increase of throughput requirement in LTE, spectrum shortage is a critical obstacle. In this regard, spectrum sharing among different wireless technologies could be a promising solution. However, this sharing approach has its own implementation hurdles. Additionally, public (license free) spectrum can be used in conjunction with licensed spectrum. In this regard, 3GPP working group is studying on the license assisted access (LAA) of LTE in the free bands [12].

$\mathrm{WiF}^{1}$ is a prominent short range communication protocol which utilizes a distributed coordination function (DCF). Its channel access mechanism performs four-way handshaking and carrier sensing [13]. The WiFi DCF mode utilizes clear channel assessment (CCA) technique for the packet transmission. The CCA includes energy detection and carrier sensing mechanism to detect the state of channel- whether it is in operation or not. WiFi node will cease transmission attempt for a random time period if the interference level crosses CCA threshold. This back-off method avoids packet collision that may happen due to coexisted LTE network transmission.

${ }^{*}$ Corresponding Author: Arif I. Sarwat, Department of Electrical and Computer Engineering, Florida International University, Miami, FL 33174, Email: asarwat@fiu.edu

${ }^{1}$ Unless or otherwise saying, IEEE $802.11 \mathrm{n}$ version will considered as WiFi in our study. 
In contrast, the LTE technology is comparatively flexible and systematic. LTE utilities dynamic scheduling for its users. The main obstacle for coexisting WiFi and LTE system in the identical band is the data transmission technique. WiFi uses CSMA/CA protocol for the transmission of OFDM. On the other hand, LTE uses the dynamic scheduling in OFDM access through which data is transmitted to several UEs simultaneously at low rate with proper time and frequency allotment [14]. LTE reserves channels to make transmission simultaneously. On the other hand, WiFi implements carrier sensing before the packet transmission. Therefore, LTE transmission will block the WiFi transmission most likely in the coexistence scenario.

Recently, 3.5 GHz band (also known as citizen broadband radio service (CBRS)) has been released for public use, which is to be shared [15, 16]. According to the guideline, the users can be classified into three classes: first tier users, second tier users, and third tier (general) users. In general, third tier/general users access the CBRS spectrum, giving priority to the first and second tier users. In several cases, third/general users can use full $150 \mathrm{MHz}$ of bandwidth in the absence of first and second tier users' activity [17]. At the worst case scenario, $80 \mathrm{MHz}$ spectrum will always be available for third tier/general users when second tier users are active and operated outside of first tier users' zones. This large amount of spectrum can provide clean channels for various wireless communication applications such as smart grid metering data communication [18, 19, 20, 21]. WiFi and LTE use the CBRS band as the potential general (third tier) users in our study.

In this study, we expand our previous work in [22], which introduces a AMI architecture based on WiFi and LTE coexistence. In the architecture, WiFi is used in smart meters for transferring data to Access point (AP). After collecting data from a group of meters, AP transfers the data to MDMS utilizing the LTE. In our framework, we consider an integrated LTE-WiFi system where LTE BS and WiFi APs are connected through IP layer. Following this, we investigate the performance of LTE-WiFi coexistence in the CBRS band considering both conventional personal mobile communication and AMI communication. For system level simulation, a time division duplexing (TDD)-LTE and $\mathrm{WiFi}$ are considered in a seven cell hexagonal layout. LTE uses a fixed duty cycle of a transmission period for its transmission, and WiFi transmits in the remaining period, in contrast. The simulation performance exhibits a harmonious coexistence relationship between WiFi and LTE. Since CRBS posses a huge chunk of free and clean spectrum, AMI based on LTE-WiFi coexistence operating in CBRS can be a potential communication resolution for smart grid. The contributions of our work is as follows:

1) We introduce a smart grid metering infrastructure based on fixed duty cycled LTE and WiFi for the first time, where LTE and WiFi shares the same spectrum band.

2) Our proposed spectrum sharing method ensures good neighborhood spectrum sharing with an option of adjusting duty of LTE transmission.

3) Our spectrum sharing technique enhances the spectral efficiency significantly.

4) We propose the usage of recently release CRBS band for metering infrastructure which can provide large amount of free and clean spectrum.

The subsequent sections are arranged as follows. The literature review on LTE-WiFi coexistence and AMI communication is discussed in Section II. The coexisted system model of LTE-WiFi coexistence in 3.5 GHz band is illustrated in Section III. Deployment scenario and performance results are illustrated in Section IV. Lastly, Section V summarizes the whole work. Some of the acronyms used in this paper are presented in Table 1

Table 1: List of Acronyms.

\begin{tabular}{|l|l|}
\hline 3GPP & 3rd Generation Partnership \\
\hline AMI & Advanced Metering Infrastructure \\
\hline AP & Access Point \\
\hline APP & application \\
\hline CSMA/CA & $\begin{array}{l}\text { Collision Sensed Multiple Access/Collision } \\
\text { Avoidance }\end{array}$ \\
\hline CCA & Clear Channel Assessment \\
\hline CBRS & Citizen Broadband Radio Service \\
\hline DCF & Decentralized Frequency Control \\
\hline EDCA & Enhanced Distributed Channel Access \\
\hline EPC & Evolved packet core \\
\hline EPC & Enhanced Packet Core \\
\hline FCC & Federal Communications Commission \\
\hline FBE & Frame Based Equipment \\
\hline GTP & GPRS tunneling protocol \\
\hline PAL & Prioritized Access License \\
\hline PHY & physical \\
\hline PDPC & packet data convergence Protocol \\
\hline GAA & General Authorized Access \\
\hline IP & Internet Layer \\
\hline LAA & Licensed Assisted Access \\
\hline LBT & Listen Before Talk \\
\hline LBE & Load Based Equipment \\
\hline LLC & logic link control \\
\hline LTE & Long Term Evolution \\
\hline M2M & Machine-to-Machine \\
\hline MDMS & Meter Data management Service \\
\hline MAC & medium access control \\
\hline OFDM & Orthogonal Frequency Division Multiplexing \\
\hline PPDU & PLCP Protocol Data Unit \\
\hline PL & Path Loss \\
\hline RLC & radio link control \\
\hline SINR & Signal-to-Interference-plus-Noise Ratio \\
\hline TPC & Transmission Power Control \\
\hline TTI & Transmission Time Interval \\
\hline UE & User Equipment \\
\hline UDP & user datagram protocol \\
\hline & \\
\hline
\end{tabular}

\section{Literature Review}

The variants of LTE working in the public/free bands can be categorized into two groups: (1) LTE-U and (2) LTE-LAA [23]. LTE-U was developed by industry consortium [24]. It uses simple mechanism and excludes modification in the air interface structure of LTE system. It is founded on the LTE release 10-12 aggregation protocol and does not embrace LBT [25]. On the other hand, LTE-LAA is based on 3rd Generation 


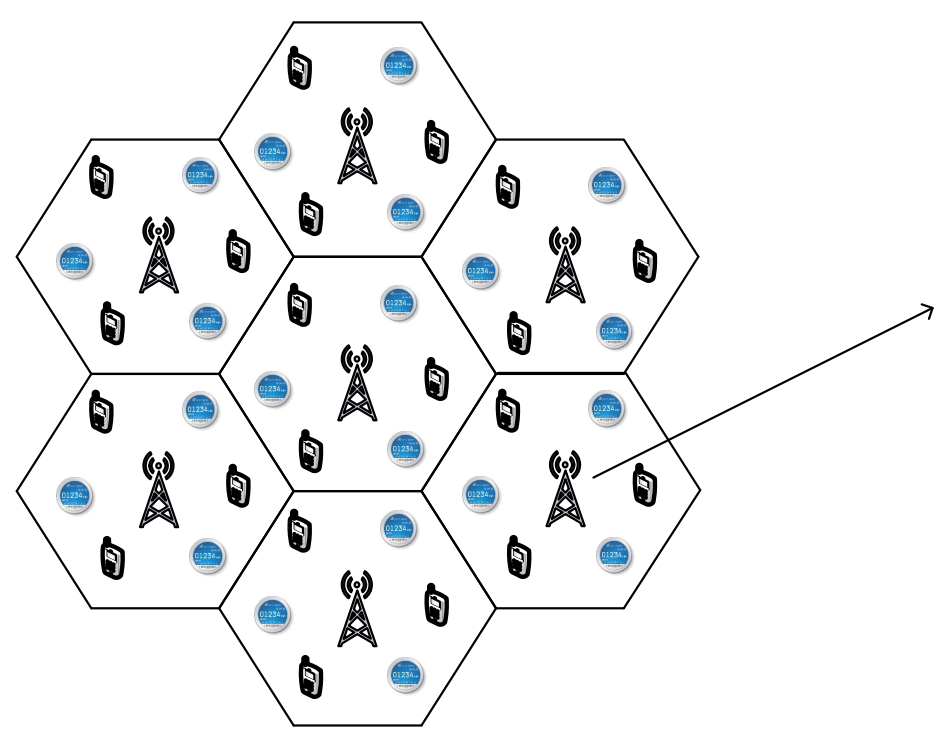

Integrated WiFi-LTE system
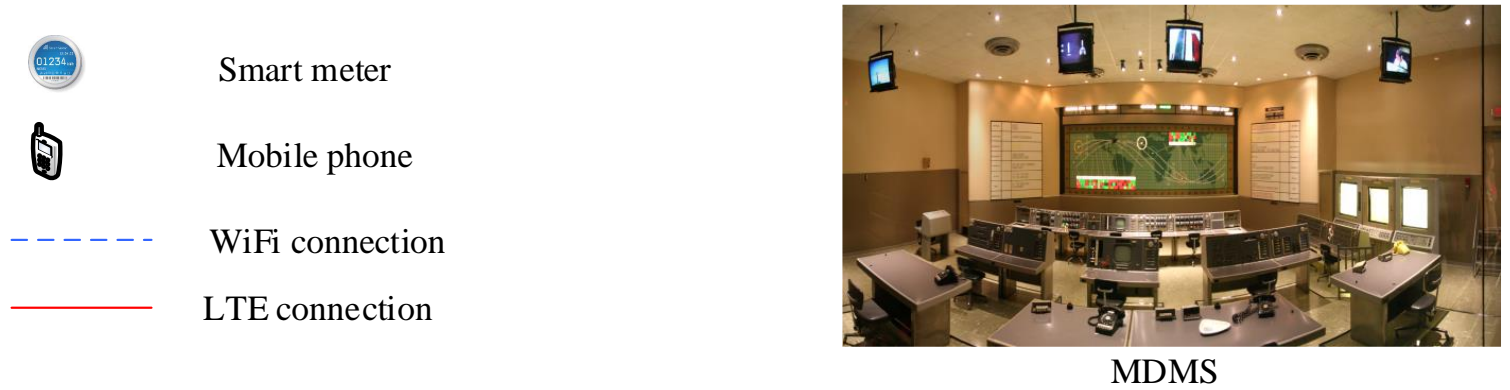

Figure 1: Architecture of smart grid metering infrastructure using LTE and WiFi on a collocated cell layout.

Partnership (3GPP) Release 13, which aims to develop a single global framework [26, 27].

In the literature, mainly three techniques have been proposed for coexistence between WiFi and LTEU/LAA. They are- 1) listen before talk (LBT);2) transmission gap; 3) dynamic channel selection. In [28], least congested channel search and adaptation of channel bandwidth are proposed for LTE 2 Qualcomm proposed an interference level based effective channel selection technique in [29]. If the interference at the operating channel crosses the threshold value, LTE alters the channel with interference measurement before and during operation at both the network and equipment side. In Japan and Europe, LBT is compulsory for data transmission in the unlicensed band. The LBT techniques can be divided into two groups- frame based equipment (FBE) and load based equipment (LBE). In FBE based LBT, a fixed slot of frame is reserved for transmission where CCA is performed [30,31]. If the channel is empty, the transmission is attempted. Otherwise, it will wait for the next frame. On the other hand, LBE based LBT is demand driven and the user equipment finds a clear channel for transmission [32, 33]. It performs extended CCA (ECCA) for clear channel access. Carrier aggression from licensed to public band is introduced in [34] using clear-to-send (CTS) and request-to-send (RTS) together with LBT. In [35], a technique of blank subframe allocation is introduced in LTE subframe, in which WiFi transmits. In [36], a identical approach is proposed, where $n$ out of 5 sub-frames of LTE is reserved for the transmission of WiFi.

In [13], the coexistence performance of hot-spot indoor scenario is explored using a semi-static system level simulator. The study found that WiFi's performance deteriorated more significantly than the performance of LTE when operated in the same band. In [37], the similar result has been found for coexistence system of ZigBee and LTE, where the performance of ZigBee is affected more compared to that of LTE. [38] explored the usage of different communication networks and recommended to use LTE for low density scenarios (i.e. rural regions) and WiFi for high density scenarios (i.e. urban regions). Meter data communication using the hybrid WiFi/LTE configuration is introduced in [39], where LTE is kept on the upper layer and WiFi in the bottom layer. However, LTE and WiFi uses different spectrum bands in this architecture and there is no spectrum sharing aspect in this study.

In our study, we introduce a fixed duty cycle based coexistence for AMI of smart grid, where LTE and WiFi shares the same spectrum band. Additionally, we consider an integrated LTE-WiFi system where LTE BS and WiFi APs are connected through IP layer. WiFi is

\footnotetext{
${ }^{2}$ Unless and otherwise specified, LTE will be considered as LTE-U or LTE-LAA throughout this study.
} 
used for meter-to-meter and meter-to-AP data communication. On the other hand, AP uses LTE to transfer data to MDMS. The duty of LTE transmission can be adjusted based on the data amount.

\section{System Architecture}

Let us assume, a coexisted network architecture consists of WiFi and LTE (LAA/LTE-U) operating in CBRS, as shown in the Fig. 1. Smart meters utilize WiFi and APs utilitie LTE for data transfer, in contrast. In addition, LTE BS and WiFi AP are attached together in the collocated environment. WiFi APs collect smart meters' data and forward them to the interconnected LTE BS. Afterwards, LTE BSs transfer data to MDMS through long range communication. Fig. 2 illustrates the protocol mapping of different components of LTE network and WiFi system. The PHY layers of WiFi $\mathrm{AP}$ and smart meters are connected together through wireless channel. Additionally, the IP layers of LTE BS and WiFi AP are integrated together in our proposed configuration. The data exchange among enhanced packet core (EPC), LTE BS, and MDMS are carried out according to standard LTE system [1].

We assume, the sets of LTE BS, WiFi STAs (i.e. smart meter), WiFi APs (i.e. collector of data from meters), and LTE UE (i.e. MDMS and other UEs) are marked as $S_{l}, U_{w}^{i}, S_{w}$, and $U_{l}^{j}$, respectively. Besides, LTE BS $j$, LTE UE/MDMS $m$, WiFi AP $i$, and meter/WiFi STA $l$ transmission power are denoted by $p_{r}^{j}, p_{r}^{m}, p_{r}^{i}$, and $p_{r}^{l}$.

The channel gain values from LTE UE $a$ to WiFi AP $j$, from WiFi STA/meter $x$ to WiFi AP $j$, from LTE BS $b(i \neq b)$ to WiFi $j$ and, from LTE BS $i$ to WiFi AP $j$ are $h_{j, r}^{a}, h_{j, r}^{x}, h_{j, r}^{b}$, and $h_{j, r}^{i}$ respectively.

During the data reception, the signal to interference plus noise ratio (SINR) of WiFi AP $j$ from meter/WiFi STA $x$ at the $r$ the resource block [40] is

$$
\operatorname{SINR}_{j, r}^{x}=\frac{h_{j, r}^{x} p_{r}^{j}}{\sum h_{j, r}^{a} p_{r}^{a}+\sum h_{j, r}^{i} p_{r}^{i}+\sum h_{j, r}^{b} p_{r}^{b}+\sigma^{2}},
$$

where $\sigma^{2}$ is the noise variance. A low SINR results poor throughput whereas high SINR ensures good throughput.

The received bit $N_{\mathrm{B}}^{x}$ at WiFi AP $j$ from WiFi STA $x$ [40] is given by

$$
N_{\mathrm{B}}^{x}=\mathrm{BT} \sum \log _{2}\left(1+\mathrm{SINR}_{j, r}^{x}\right),
$$

where $\mathrm{B}$ and $\mathrm{T}\left(\mathrm{T}=\sum r\right)$ are the bandwidth and transmission time, respectively. The received bit number is dependent on the SINR value.

The throughput of WiFi STA/meter $x$ during the up link (UL) can be expressed [40] as

$$
C^{x}=\frac{N_{\mathrm{B}}^{x}}{T_{\mathrm{tx}}+T_{\mathrm{wait}}},
$$

where $T_{\text {wait }}$ and $T_{\text {tx }}$ represent the wait time and transmission time of $\mathrm{WiFi}$, respectively.

For down-link capacity calculation, similar equations: (1)-(3) are applicable.

The arrival rate of traffic for both WiFi and LTE is $\lambda$. The function relating delay of incoming packets (d) [40] is then

$$
f(d)=\lambda e^{\lambda d} .
$$

\section{Deployment Scenario and Simu- lation Results}

As illustrated in Fig. 1. a coexisted network layout of 7 cells is considered to investigate the system performance. A Matlab simulator founded on 3GPP standard was used for simulation similar to [13, 41]. For each integrated WiFi AP and LTE BS, 10 LTE UEs and 10 smart meters (WiFi STAs) are dropped randomly in each cell. One of the 10 LTE UEs is used as the MDMS. For both WiFi and LTE, the data arrival rate is kept same as $\lambda_{\mathrm{WiFi}}=\lambda_{\mathrm{LTE}}=2.5$ packets $/$ second. The PHY and MAC layer of IEEE $802.11 \mathrm{n}$ and LTE are enforced in the simulation scenario. Single UE is scheduled for DL/UL during a transmission time interval (TTI) and the corresponding SINR is sent to the BS. During one subframe of transmission, bandwidth is divided among all UEs based on request and waiting LTE UEs. TABLE 2 summarizes the simulation parameter for LTE, where values were chosen according to 3GPP LTE standard [12].

Enhanced distributed channel access (EDCA) and advanced clear channel assessment (CCA) have been enforced for WiFi channel access mechanism i.e. CSMA/CA. After receiving a beacon signal, all WiFi STAs (i.e. meters) with traffic will be in competition for accessing channel. Data transmission or reception will be at postponed without receiving a beacon signal.

Table 2: PHY and MAC Layer Parameters for LTE.

\begin{tabular}{ll}
\hline Parameter & Value \\
\hline Frequency band & $3.5 \mathrm{GHz}$ \\
$\begin{array}{l}\text { Bandwidth } \\
\text { Transmission power of DL trans- } \\
\text { mission }\end{array}$ & $20 \mathrm{MHz}$ \\
$\begin{array}{l}\text { Velocity of UEs } \\
\text { Transmission power of UL trans- }\end{array}$ & $0 \mathrm{dL}$ \\
mission & PL Based TPC \\
Frame duration & $10 \mathrm{~ms}$ \\
Type of scheduling & Round Robin \\
$P_{0}$ & $-106 \mathrm{dBm}$ \\
TTI & $1 \mathrm{~ms}$ \\
Packet arrival rate $(\lambda)$ & 2.5 \\
\hline
\end{tabular}

The WiFi STA will sense for a free channel before any kind of transmission. Transmission will take place only if the channel is in idle, otherwise it will back off. After a randomly chosen back off time, next transmission 


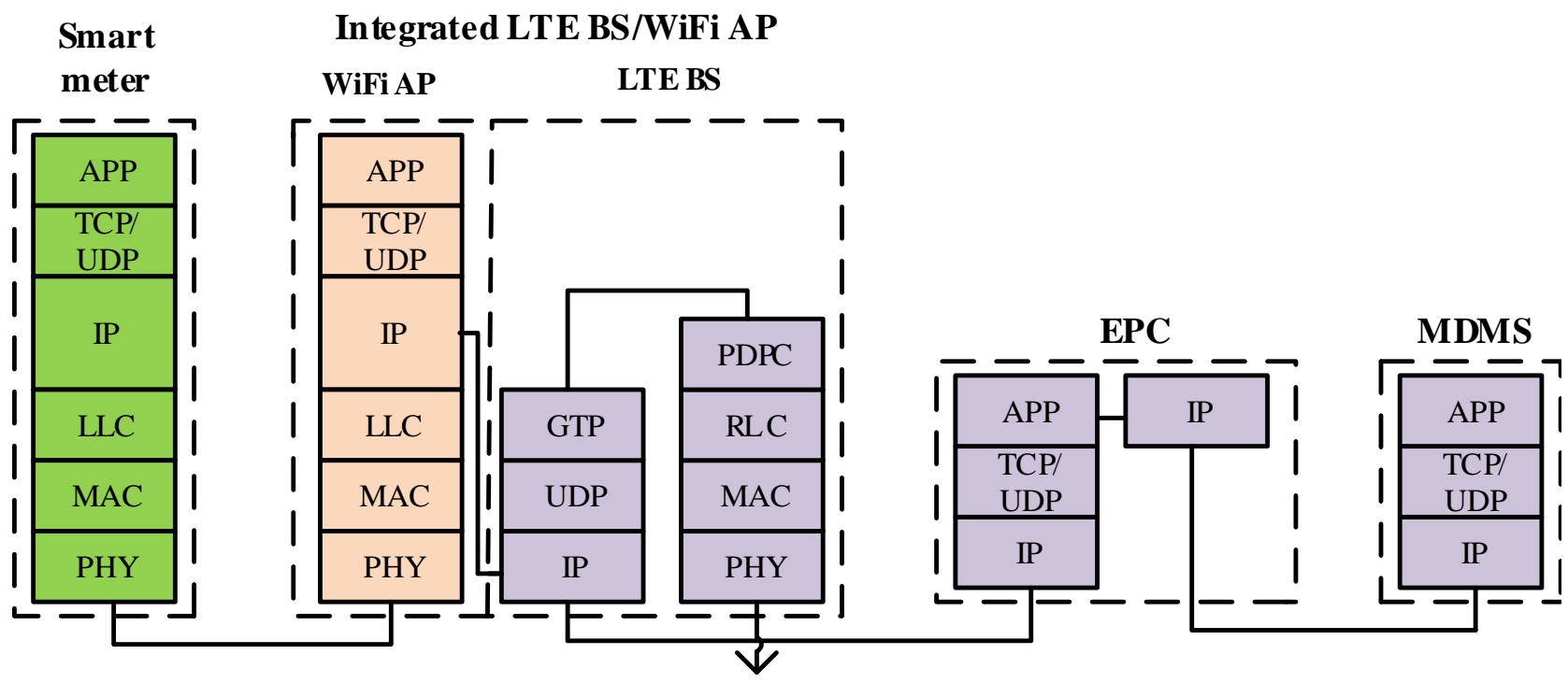

Figure 2: Protocol mapping among various entities of LTE and WiFi system.

will be attempted. TABLE 3 summarizes the WiFi simulation parameter used in the simulation [13, 18, 42].

The abstract of PHY layer is used for calculating Shannon capacity of LTE and WiFi at the $4 \mu$ s granularity of WiFi OFDM symbol period. FTP traffic model-2 is applied for both LTE and WiFi traffic [43]. In this study, duty cycles- $20 \%, 40 \%, 60 \%$ and $80 \%$ of $50 \mathrm{~ms}$ time period are utilized for LTE transmission and the rest of $50 \mathrm{~ms}$, i.e. $80 \%, 60 \%, 40 \%$ and $20 \%$ are used for WiFi transmission, respectively. The data rate performance of coexisting WiFi and LTE system is presented in Table 4 For $20 \%$ duty cycle, the LTE throughput is $10.3 \mathrm{Mbps}$ and the throughput of WiFi is $155.2 \mathrm{Mpbs}$.

Table 3: PHY and MAC Layer Parameters for WiFi.

\begin{tabular}{ll}
\hline Parameter & Value \\
\hline Frequency band & $3.5 \mathrm{GHz}$ \\
$\begin{array}{l}\text { Bandwidth } \\
\text { Transmission power of Down- }\end{array}$ & $20 \mathrm{MHz}$ \\
link/Uplink & $23 \mathrm{dBm}$ \\
Velocity of STA/meter & \\
Category of access & $0 \mathrm{~ms}$ \\
Protocol for MAC layer & Best Effort \\
Sensing threshold of CCA & EDCA \\
Energy detection threshold of CCA & $-82 \mathrm{dBm}$ \\
Number of PPDU service bits & $-65 \mathrm{dBm}$ \\
Number of PPDU tail bits & $16 \mathrm{bits}$ \\
Window size for contention & $12 \mathrm{bits}$ \\
Noise figure & $\mathcal{U}(0,31)$ \\
Interval for beacon transmission & 6 \\
Threshold of symbol detection in & $100 \mathrm{~ms}$ \\
OFDM & $10 \mathrm{~dB}$ \\
Threshold of beacon error ratio & 15 \\
Arrival rate of packets $(\lambda)$ & 2.5 \\
\hline
\end{tabular}

For $40 \%$ duty cycle of LTE, the throughput of LTE and WiFi are $18.8 \mathrm{Mbps}$ and $111.78 \mathrm{Mbps}$, respectively. For $60 \%$ duty cycle of LTE, the throughput of LTE and WiFi are $36.3 \mathrm{Mbps}$ and $36.1 \mathrm{Mpbs}$, respectively. The throughput of LTE is boosted to $38.6 \mathrm{Mbps}$ after increasing the duty cycle of LTE to $80 \%$. However, WiFi capacity is reduced to $31.2 \mathrm{Mbps}$. Therefore, for increment of LTE transmission duty cycle, the LTE capacity is improved and WiFi is degraded drastically. The reason behind the WiFi throughput degradation is the increased transmission back off on the extended period of LTE transmission.

The energy efficiency (EE) performance of coexisted systems is demonstrated in Table 5 It is noted that the EE of LTE is improved with the increment of duty cycle of LTE. The EEs of LTE at $20 \%$ and $80 \%$ duty cycle are $3.32 \times 10^{8}$ bits/joule and $1.245 \times 10^{9}$ bits/joule, respectively. On the other hand, the EE of WiFi is degraded with the increase of LTE duty cycle. The EEs of WiFi at $20 \%$ and $80 \%$ duty cycle of LTE are $7.76 \times 10^{8}$ bits/joule and $1.56 \times 10^{8}$ bits/joule, respectively. More significantly, the overall $\mathrm{EE}$ of the coexisted system continues to improve with the increment of LTE transmission duty cycle. The overall $\mathrm{EE}$ is boosted from $1.008 \times 10^{9}$ bits/joule to $1.401 \times 10^{9}$ bits/joule. This reflects a good neighborhood relationship between LTE and WiFi regardless of degradation of overall throughput of the coexisted system.

The SINR distribution of coexisting LTE and WiFi system is illustrated in Fig. 3. For 20\% and 40\% duty cycle of LTE transmission, WiFi has better SINR distribution over LTE system. This is reflected in Fig. 3(a) and Fig. 3(b). For the increment of LTE duty cycle to $60 \%$ and $80 \%$, the SINR of LTE system improves while the SINR of WiFi degrades consequently. This is demonstrated in Fig. 3(c) and Fig. 3(d), respectively.

In urban or suburban areas, large number of smart meters will use WiFi for sending consumption data to $\mathrm{AP}$, and later the collected data will be sent to MDMS using LTE. Therefore, more opportunity of accessing channel by WiFi is desirable in this case. In this regard, $20 \%$ and $40 \%$ duty cycle of LTE transmission can be prudent choice for AMI infrastructure. On the other 
Table 4: Capacity of the coexisted LTE-WiFi system

\begin{tabular}{|l|l|l|l|l|l|l|}
\hline \multirow{2}{*}{ Duty cycle } & \multicolumn{3}{|c|}{ LTE } & \multicolumn{3}{c|}{ WiFi } \\
& $\begin{array}{l}\text { Down link } \\
\text { (bits/second) }\end{array}$ & $\begin{array}{l}\text { Up link } \\
\text { (bits/second) }\end{array}$ & $\begin{array}{l}\text { Total } \\
\text { (bits/second) }\end{array}$ & $\begin{array}{l}\text { Down link } \\
\text { (bits/second) }\end{array}$ & $\begin{array}{l}\text { Up link } \\
\text { (bits/second) }\end{array}$ & $\begin{array}{l}\text { Total } \\
\text { (bits/second) }\end{array}$ \\
\hline $20 \%$ & $9.15 \times 10^{6}$ & $1.153 \times 10^{5}$ & $1.030 \times 10^{7}$ & $8.343 \times 10^{7}$ & $7.176 \times 10^{7}$ & $1.552 \times 10^{8}$ \\
\hline $40 \%$ & $1.667 \times 10^{7}$ & $2.17 \times 10^{6}$ & $1.884 \times 10^{7}$ & $6.435 \times 10^{7}$ & $4.742 \times 10^{7}$ & $1.1178 \times 10^{8}$ \\
\hline $60 \%$ & $2.81 \times 10^{7}$ & $8.16 \times 10^{6}$ & $3.63 \times 10^{7}$ & $2.45 \times 10^{7}$ & $1.16 \times 10^{7}$ & $3.61 \times 10^{7}$ \\
\hline $80 \%$ & $2.71 \times 10^{7}$ & $1.15 \times 10^{7}$ & $3.86 \times 10^{7}$ & $1.66 \times 10^{7}$ & $1.46 \times 10^{7}$ & $3.12 \times 10^{7}$ \\
\hline
\end{tabular}

Table 5: Energy efficiency performance of coexisted LTE-WiFi system

\begin{tabular}{|c|c|c|c|}
\hline Duty cycle & LTE (bits/joule) & WiFi (bits/joule) & Total (bits/joule) \\
\hline $20 \%$ & $3.32 \times 10^{8}$ & $7.76 \times 10^{8}$ & $1.008 \times 10^{9}$ \\
\hline $40 \%$ & $6.07 \times 10^{8}$ & $5.58 \times 10^{8}$ & $1.17 \times 10^{9}$ \\
\hline $60 \%$ & $1.171 \times 10^{9}$ & $1.80 \times 10^{8}$ & $1.351 \times 10^{9}$ \\
\hline $80 \%$ & $1.245 \times 10^{9}$ & $1.56 \times 10^{8}$ & $1.401 \times 10^{9}$ \\
\hline
\end{tabular}

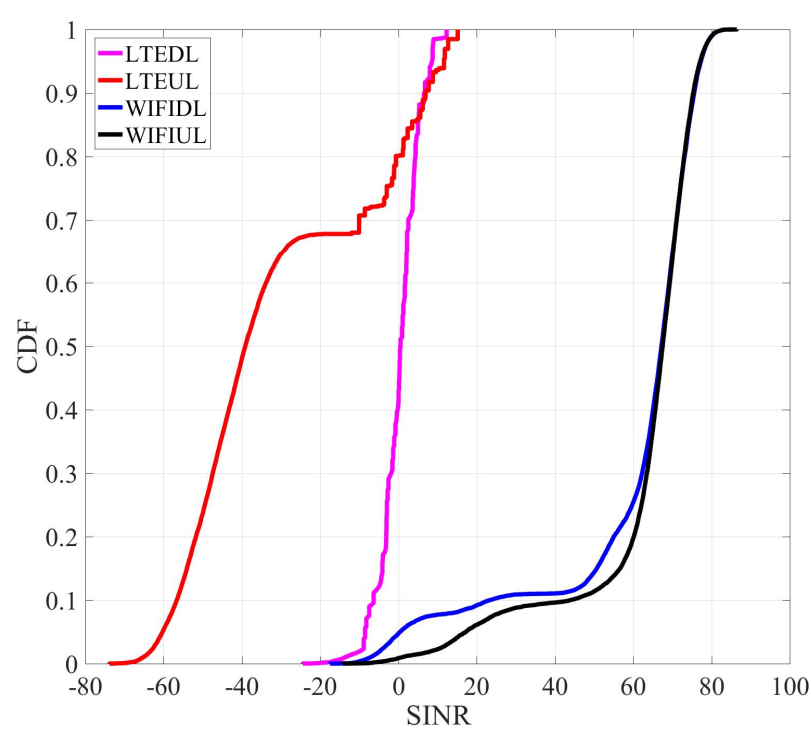

(a)

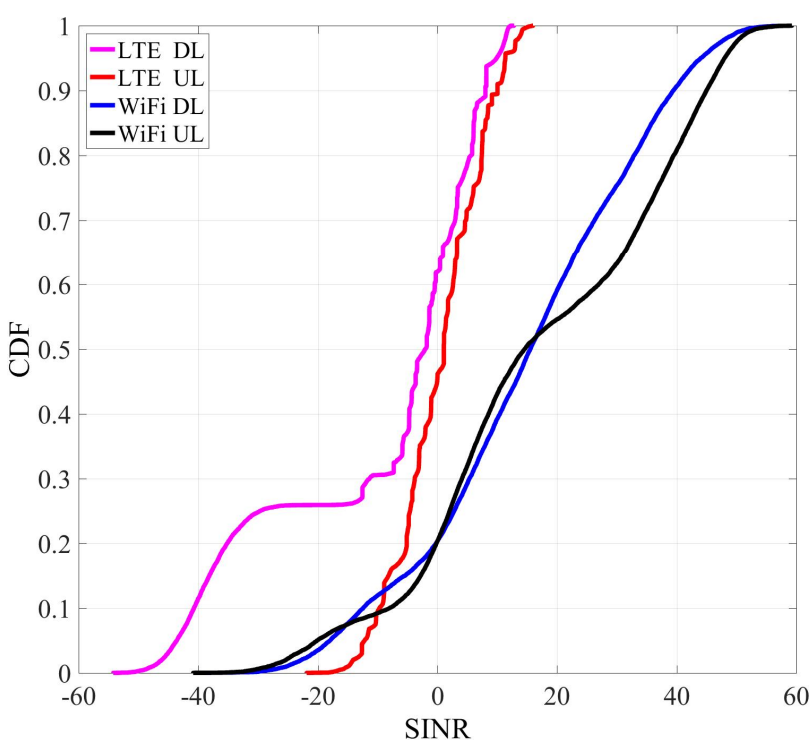

(c)

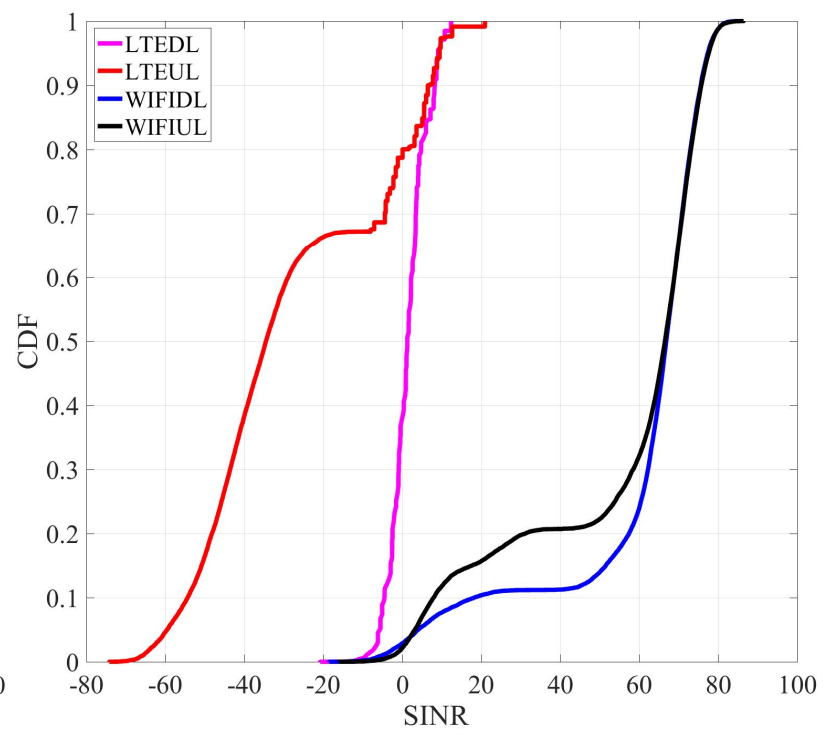

(b)

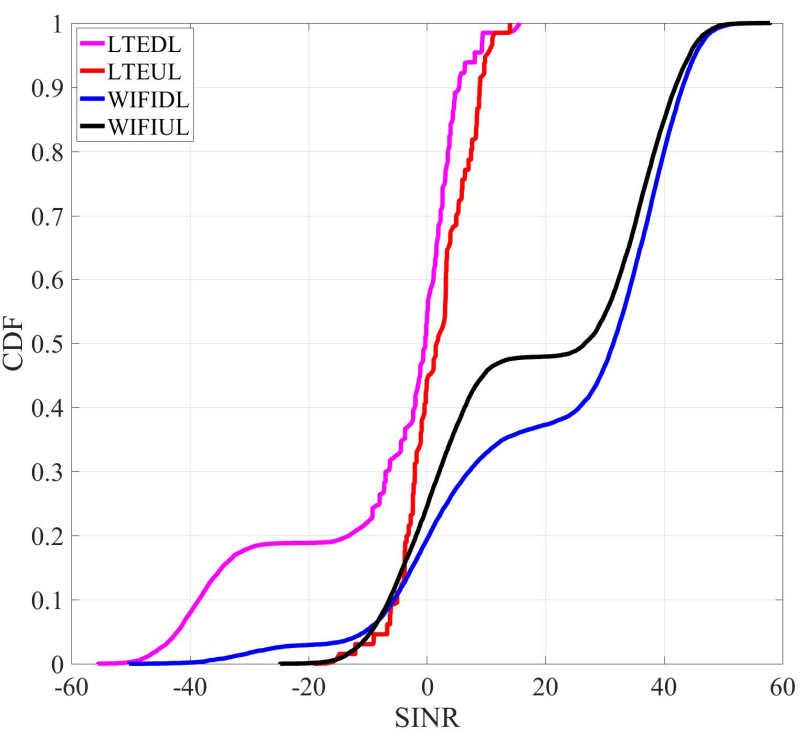

(d)

Figure 3: SINR distribution of coexisted LTE-WiFi system (a) SINR distribution at $20 \%$ duty cycle (b) SINR distribution at $40 \%$ duty cycle (c) SINR distribution at $60 \%$ duty cycle (d) SINR distribution at $80 \%$ duty cycle 
hand, in the rural areas, scattered and limited number of meters will use WiFi. Therefore, in this case, more access can be given to LTE by selecting higher duty cycles such as $60 \%$ and $80 \%$.

\section{Conclusion}

In this study, a collocated WiFi and LTE based advance metering infrastructure is proposed for smart grid. For meter-to-meter data communication, WiFi is proposed. On the other hand, for sending collected data from a group of meters to MDMS, LTE is proposed. A fixed duty cycle of a transmission time is reserved for LTE and the rest of the period is given to $\mathrm{WiFi}$ system. The simulation performance shows a harmonious neighborhood spectrum sharing between LTE and WiFi. With the increase of LTE duty cycle, the throughput, energy efficiency and SINR of LTE are improved along with degradation of those of WiFi.

The transmission duty cycle of LTE is adjustable based on the amount of data and number of smart meters. In particular, lower duty cycle of LTE transmission can be selected for urban and suburban areas where the density of smart meters are high and meters need more access to WiFi. On the other hand, higher duty of LTE transmission can be selected for rural areas where the density of smart meter is low. The CBRS band has a big amount of free, underutilized, and clean spectrum for wireless network. So, network consists of coexisting LTE and WiFi in CBRS band can be a viable communication solution for the metering infrastructure of smart grid.

Acknowledgment The work is a outcome of the research supported by the U.S. National Science Foundation under the grant CPS-1446570 and CAREER1553494.

\section{References}

[1] A. F. Molisch, 3GPP LongTerm Evolution. Wiley-IEEE Press, 2011, pp. 665-698. [Online]. Available: http://ieeexplore.ieee. org/xpl/articleDetails.jsp?arnumber $=5635449$

[2] I. Parvez, M. Jamei, A. Sundararajan, and A. I. Sarwat, "RSS based loop-free compass routing protocol for data communication in advanced metering infrastructure (AMI) of Smart Grid," in 2014 IEEE Symposium on Computational Intelligence Applications in Smart Grid (CIASG), Dec 2014, pp. 1-6.

[3] I. Parvez, A. I. Sarwat, J. Pinto, Z. Parvez, and M. A. Khandaker, "A gossip algorithm based clock synchronization scheme for smart grid applications," CoRR, vol. abs/1707.08216, 2017. [Online]. Available: http://arxiv.org/abs/1707.08216

[4] M. Jafari, T. O. Olowu, and A. I. Sarwat, "Optimal smart inverters volt-var curve selection with a multi-objective volt-var optimization using evolutionary algorithm approach," in 2018 North American Power Symposium (NAPS), Sep. 2018, pp. 1-6.

[5] I. Parvez, A. Islam, and F. Kaleem, "A key management-based two-level encryption method for ami," in 2014 IEEE PES General Meeting - Conference Exposition, July 2014, pp. 1-5.

[6] I. Parvez, A. I. Sarwat, L. Wei, and A. Sundararajan, "Securing metering infrastructure of smart grid: A machine learning and localization based key management approach," Energies, vol. 9, no. 9, 2016.
[7] I. Parvez, F. Abdul, and A. I. Sarwat, "A location based key management system for advanced metering infrastructure of smart grid," in 2016 IEEE Green Technologies Conference (GreenTech), April 2016, pp. 62-67.

[8] M. O. Onibonoje and T. O. Olowu, "Real-time remote monitoring and automated control of granary environmental factors using wireless sensor network," in 2017 IEEE International Conference on Power, Control, Signals and Instrumentation Engineering (ICPCSI), Sep. 2017, pp. 113-118.

[9] T. O. Olowu, M. Jafari, and A. I. Sarwat, "A multi-objective optimization technique for volt-var control with high pv penetration using genetic algorithm," in 2018 North American Power Symposium (NAPS), Sep. 2018, pp. 1-6.

[10] I. Parvez, A. Sundararajan, and A. I. Sarwat, "Frequency band for HAN and NAN communication in Smart Grid," in Proc. IEEE Symp. on Comp. Intel. App. in Smart Grid (CIASG), Dec 2014, pp. 1-5.

[11] I. Parvez, A. Rahmati, I. Güvenç, A. I. Sarwat, and H. Dai, "A Survey on Low Latency Towards 5G: RAN, Core Network and Caching Solutions," CoRR, vol. abs/1708.02562, 2017. [Online]. Available: http://arxiv.org/abs/1708.02562

[12] 3GPP, "Study on Licensed-Assisted Access using LTE," RP141397, Tech. Rep., Sep. 2014.

[13] N. Rupasinghe and İ. Güvenç, "Licensed-assisted access for WiFi-LTE coexistence in the unlicensed spectrum," in Proc. IEEE Glob. Work. (GC Wkshps). IEEE, 2014, pp. 894-899.

[14] F. Chaves, A. Cavalcante, E. Almeida, F. Abinader Jr, R. Vieira, S. Choudhury, and K. Doppler, "LTE/Wi-Fi coexistence: Challenges and mechanisms," in 2013, XXXI SIMPOSIO BRASILEIRO DE TELECOMUNICACOES-SBrT2013, 2013.

[15] FCC, "Amendment of the Commissions Rules with Regard to Commercial Operations in the 3550-3650 MHz Band," Report and Order and Second Further Notice of Proposed Rulemaking, Apr. 2015.

[16] L. Stefani, "The FCC Raises the Curtain on the Citizens Broadband Radio Service," CommLawBlog Article, May 2015. [Online]. Available: http://www.commlawblog.com/2015/05/ articles/unlicensed-operations-and-emer/the-fcc-raises-thecurtain-on-the-citizens-broadband-radio-service/

[17] G. Locke and L. E. Strickling, "An assessment of the near-term viability of accommodating wireless broadband systems in the $1675-1710 \mathrm{MHz}, 1755-1780 \mathrm{MHz}, 3500-3650 \mathrm{MHz}$, and $4200-4220 \mathrm{MHz}, 4380-4400 \mathrm{MHz}$ bands," Report, Oct. 2010. [Online]. Available: http://www.ntia.doc.gov/files/ntia/ publications/fasttrackevaluation11152010.pdf

[18] I. Parvez, M. Sriyananda, İ. Güvenç, M. Bennis, and A. Sarwat, "Cbrs spectrum sharing between lte- $u$ and wifi: A multiarmed bandit approach," Mobile Information Systems, vol. 2016, 2016.

[19] S. Rahman, M. Moghaddami, A. I. Sarwat, T. Olowu, and M. Jafaritalarposhti, "Flicker estimation associated with pv integrated distribution network," in SoutheastCon 2018, April 2018, pp. 1-6.

[20] I. Parvez, N. Chotikorn, and A. I. Sarwat, "Average quantized consensus building by gossip algorithm using 16 bit quantization and efficient data transfer method."

[21] Y. Mekonnen, M. Haque, I. Parvez, A. H. Moghadasi, and A. I. Sarwat, "LTE and wi-fi coexistence in unlicensed spectrum with application to smart grid: A review," CoRR, vol. abs/1708.09005, 2017. [Online]. Available: http://arxiv.org/abs/1708.09005

[22] I. Parvez, T. Khan, A. I. Sarwat, and Z. Parvez, "Laa-lte and wifi based smart grid metering infrastructure in 3.5 ghz band," in 2017 IEEE Region 10 Humanitarian Technology Conference (R10-HTC), Dec 2017, pp. 151-155.

[23] X. Wang, S. Mao, and M. X. Gong, "A survey of lte wi-fi coexistence in unlicensed bands," GetMobile: Mobile Computing and Communications, vol. 20, no. 3, pp. 17-23, 2017.

[24] A. Babaei, J. Andreoli-Fang, Y. Pang, and B. Hamzeh, "On the impact of LTE-U on Wi-Fi performance," International Journal of Wireless Information Networks, vol. 22, no. 4, pp. 336-344, 2015. 
[25] M. Sriyananda, I. Parvez, I. Güvene, M. Bennis, and A. I. Sarwat, "Multi-armed bandit for LTE-U and WiFi coexistence," in Proc. IEEE Wire. Commun. and Netw. Conf. IEEE, 2016, pp. 1-6.

[26] G. RP-140808, "Review of regulatory requirements for unlicensed spectrum)," AlcatelLucent, Alcatel-Lucent Shanghai Bell, Ericsson, Huawei, HiSilicon, IAESI, LG, Nokia, NSN, Qualcomm, NTT Docomo, Tech. Rep., June 2014.

[27] Nokia, "Nokia LTE for unlicensed spectrum," AlcatelLucent, Alcatel-Lucent Shanghai Bell, Ericsson, Huawei, HiSilicon, IAESI, LG, Nokia, NSN, Qualcomm, NTT Docomo, Tech. Rep., June 2014.

[28] F. M. Abinader, E. P. L. Almeida, F. S. Chaves, A. M. Cavalcante, R. D. Vieira, R. C. D. Paiva, A. M. Sobrinho, S. Choudhury, E. Tuomaala, K. Doppler, and V. A. Sousa, "Enabling the coexistence of LTE and Wi-Fi in unlicensed bands," IEEE Commun. Mag., vol. 52, no. 11, pp. 54-61, Nov. 2014.

[29] Qualcomm, "Qualcomm Research LTE in Unlicensed Spectrum: Harmonious Coexistence with WiFi," 3GPP RAN1 standard contribution, Tech. Rep., Jun. 2014.

[30] ZTE, "Frame structure design for LAA considering LBT," 3GPP TSG RAN WG1 Meeting no.80, Feb. 2015.

[31] R. Zhang, M. Wang, L. X. Cai, X. Shen, L.-L. Xie, and Y. Cheng, "Modeling and analysis of MAC protocol for LTE-U co-existing with Wi-Fi," in Proc. IEEE Glob. Commun. Conf. (GLOBECOM). IEEE, 2015, pp. 1-6.

[32] O. El-Samadisy, M. Khedr, and A. El-Helw, "Performance Evaluation of MAC for IEEE 802.11 and LAA LTE," in Proc. Intern. Conf. on Comput. Science and Comput. Intell. (CSCI), Dec 2016, pp. $923-928$.

[33] A. k. Ajami and H. Artail, "Fairness in future licensed assisted access (laa) lte networks: What happens when operators have different channel access priorities?" in Proc. IEEE Intern. Conf. on Commun. Works. (ICC Workshops), May 2017, pp. 67-72.

[34] R. Ratasuk, M. A. Uusitalo, N. Mangalvedhe, A. Sorri, S. Iraji, C. Wijting, and A. Ghosh, "License-exempt LTE deployment in heterogeneous network," in Int. Symp. on Wireless Commun. Sys., Paris, France, Aug. 2012, pp. 246-250.
[35] E. Almeida, A. M. Cavalcante, R. C. D. Paiva, F. S. Chaves, F. M. Abinader, R. D. Vieira, S. Choudhury, E. Tuomaala, and K. Doppler, "Enabling LTE/WiFi coexistence by LTE blank subframe allocation," in Proc.IEEE Int. Conf., Budapest, Hungary, Jun. 2013, pp. 5083-5088.

[36] T. Nihtil, V. Tykhomyrov, O. Alanen, M. A. Uusitalo, A. Sorri, M. Moisio, S. Iraji, R. Ratasuk, and N. Mangalvedhe, "System performance of LTE and IEEE 802.11 coexisting on a shared frequency band," in Proc. IEEE Wireless Commun. and Net. Conf.(WCNC), Shanghai, China, Apr. 2013, pp. 1038-1043.

[37] I. Parvez, N. Islam, N. Rupasinghe, A. I. Sarwat, and . Gven, "LAA-based LTE and ZigBee coexistence for unlicensed-band smart grid communications," in Proc. IEEE SoutheastCon conf., March 2016, pp. 1-6.

[38] F. Granelli, D. Domeniconi, N. L. S. D. Fonseca, and B. Tsetsgee, "On the usage of wifi and lte for the smart grid," in 2014 7th International Conference on Ubi-Media Computing and Workshops, July 2014, pp. 1-5.

[39] F. Koohifar, N. Saputro, I. Guvenc, and K. Akkaya, "Hybrid wi-fi/lte aggregation architecture for smart meter communications," in 2015 IEEE International Conference on Smart Grid Communications (SmartGridComm), Nov 2015, pp. 575-580.

[40] N. Rupasinghe and . Gven, "Licensed-assisted access for wifi-lte coexistence in the unlicensed spectrum," in 2014 IEEE Globecom Workshops (GC Wkshps), Dec 2014, pp. 894-899.

[41] I. Parvez, N. Islam, N. Rupasinghe, A. I. Sarwat, and . Gven, "LAA-based LTE and ZigBee coexistence for unlicensed-band smart grid communications," in SoutheastCon 2016, March 2016, pp. 1-6.

[42] M. G. S. Sriyananda, I. Parvez, I. Gvene, M. Bennis, and A. I. Sarwat, "Multi-armed bandit for LTE-U and WiFi coexistence in unlicensed bands," in 2016 IEEE Wireless Communications and Networking Conference, April 2016, pp. 1-6.

[43] 3GPP, "Evolved Universal Terrestrial Radio Access (E-UTRA); Further advancements for E-UTRA physical layer aspects (Release 9)," 3GPP Technical Report, Tech. Rep., Mar. 2010. 